\title{
Uso de esteroides en la ruptura prematura de membranas pretérmino
}

\author{
María Cristina Barco Burgos*
}

RESUMEN: Se hace un corto recuento sobre la forma como se descubrió el surfactante, el desarrollo morfológico del pulmón fetal y la composición del surfactante. Se pasa luego a enunciar lo que se conoce sobre la acción de los esteroides en la maduración pulmonar y los posibles efectos adversos a éstos sobre el feto y el neonato.

Con el objeto de tener elementos de juicio que permitan concluir sobre el efecto benéfico o no de los esteroides sobre la maduración pulmonar en pacientes con R.P.M. pretérmino, se hace un resumen de los principales trabajos que se han efectuado en los últimos años sobre este aspecto.

PALABRAS CLA VES:Esteroides, maduración pulmonar, ruptura prematura de membranas pretérmino, membrana hialina, infección.

SUMMARY: This paper starts with a short summary about the discovery of the surfactant, the morphologic development of the fetal lung and the composition of the surfactant. It explains what is known about the effect of antenatal steroid therapy on the fetal lung and the possible adverse effects of them. In order to achieve conclusions about the beneficial effects or not of the steroids, a summary of the most important investigations during the last years in made.

KEY WORDS: Steroids, preterm premature rupture of membranes, lung maturation, hialine membrane, infection.

\section{Breve recuento histórico}

Los signos y síntomas del síndrome de dificultad respiratoria (SDR) fueron descritos por primera vez por Hochein en 1903, quien observó un revestimiento algodonoso en los pulmones de dos niños que murieron poco después del nacimiento. Esto condujo posteriormente a la aparición del término "enfermedad de la membrana hialina". En 1929 Von Noergrand comenzó a hablar de la tensión superficial en la interfase aire-tejido del alvéolo, y solo hasta 1957 Clements encontró que existía una sustancia que disminuía la tensión superficial en los extractos salinos del material de lavado pulmonar. Posteriormente se demostró que los componentes activos de superficie de los alvéolos son atribuibles a las propiedades de un complejo lipoproteico llamado surfactante. Avery y Mead en 1959 fueron los primeros en señalar que el síndrome de sufrimiento respiratorio está causado por un déficit en la biosíntesis del surfactante en el pulmón del feto y del neonato. En 1961 Klaus y Cols. demostraron que el principal componente activo de superficie del surfactante era atribuible a una lecitina específica, la dipalmitoilfosfatidil colina. Se supo luego que el surfactante se forma específicamente en los neumocitos tipo II que recubren el alvéolo.

\section{Desarrollo morfológico del pulmón fetal}

Consta de tres etapas:

1. Etapa glandular. Va desde la concepción hasta la semana 16 de gestación. Se caracteriza por la presencia

\footnotetext{
* Profesora Departamento de Obstetricia y Ginecología. Facultad de Medicina. Universidad de Antioquia.
}

de una masa laxa de tejido mesenquimatoso rodeando yemas de células endodérmicas.

2. Etapa canalicular: Se inicia desde la semana 16 y va hasta la semana 24 de gestación. El tejido endodérmico crece y se ramifica para formar bronquiolos primitivos. Empieza también la vascularización del epitelio.

3. Etapa del saco terminal: va desde las 24 semanas hasta el término. Ocurre mayor división de bronquiolos de los cuales surgen sáculos de pared delgada. A las 26 semanas, el epitelio está suficientemente especializado para permitir el intercambio gaseoso habiendo empezado entonces la potencialidad de viabilidad.

El crecimiento subsiguiente se caracteriza por el desarrollo de los alvéolos como resultado de la diferenciación del epitelio respiratorio en células tipo I y tipo II (fig. 1).

Las células tipo I son planas de soporte sin función secretoria conocida.

Las tipo II son más grandes, redondas y ocupan las áreas de unión entre los alvéolos. Son las encargadas de producir el surfactante.

La aparición de cuerpos laminares se ve por primera vez en estas células a las 26 semanas de gestación y la diferenciación de las células tipo II se completa usualmente a las 28 semanas. La maduración posterior se acompaña de aumento del número de cuerpos de inclusión y disminución del contenido de glicógeno de las células tipo II. Estudios con microscopio electrónico han revelado actividad secretoria tan tempranamente como a las 26-27 semanas (1).

\section{Surfactante}

El surfactante es un término genérico que se refiere a un grupo de "detergentes" responsables de disminuir la 
Figura 1

EXTENSION DEL SURFACTANTE POR LA SUPERFICIE ALVEOLAR: REPRODUCCION TRIDIMENSIONAL

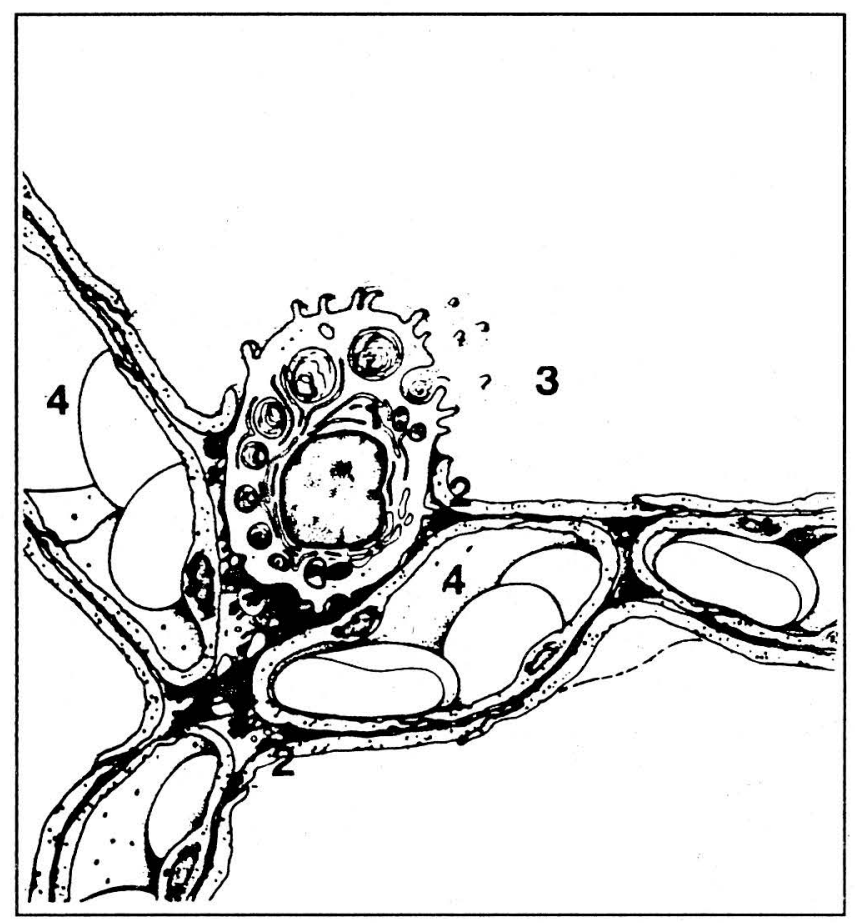

El surfactante sintetizado por los neumocitos tipo Il y liberado en la superficie alveolar se extiende como una película monomolecular sobre el epitelio alveolar. El surfactante cubre las desigualdades de nivel entre los neumocitos de tipos I y II. Se observan microvellos aislados que afloran al espacio alveolar a través de la película de surfactante.

$1=$ Neumocito tipo II.

2 = Neumocito tipo I.

3 = Película de surfactante.

$4=$ Capilares alveolares.

tensión superficial dentro del alvéolo. Está compuesto por $90 \%$ de lípidos, $10 \%$ de proteínas y a $2 \%$ con fosfolípidos de los cuales el $70 \%$ corresponde a fosfatidil colina o lecitina. Los otros fosfolípidos son el fosfatidilglicerol (10.15\%), fosfatidilinositol (5-10\%), la fosfatidil etanolamina, la fosfatidil serina y esfingomielina -que es en realidad un esfingolípido-.

La primera lecitina que parece en el ser humano es la $\alpha$ palmitoil, $B$ miristoil-fosfatidil colina y posteriormente, a partir de la semana 35 , aparece la $\alpha, \beta$-dipalmitoilfosfatidil-colina, un compuesto disaturado que es la molécula activa de superficie. La saturación se logra por medio de la enzima acyltransferasa.

La secreción del surfactante empieza a la semana 26 de gestación, y hasta la semana 30-32 la esfingomielina es mayor que la lecitina. En la semana 32 la relación L/S es más o menos de uno y de ahí en adelante la lecitina empieza a exceder a la esfingomielina acelerándose rápidamente entre la semana 34-36. Al mismo tiempo la proporción de lecitina disaturada también está aumentada y los fosfolípidos ayudadores (fosfatidilglicerol y fosfatidil inositol) contribuyen para crear un detergente más efectivo. Como la efectividad del surfactante depende de una interacción compleja de todos los fosfolípidos, la enfermedad de la membrana hialina puede presentarse no sólo a partir de una deficiencia de la producción, sino también por un producto cualitativamente defectuoso (1).

La madurez funcional pulmonar fetal se alcanza, en situación de normalidad, alrededor de la semana 35 de gestación, traduciéndose en el líquido amniótico mediante un incremento muy marcado de las sustancias surfactantes. Esto es el reflejo de la puesta en marcha de la vía de la fosfocolina-transferasa a nivel del alvéolo, sintetizando una mayor cantidad y calidad de lecitina saturada con ácido palmítico (2).

Usualmente la maduración pulmonar es considerada como sinónimo del desarrollo de las células alveolares tipo II y la síntesis y secreción de surfactante. Sin embargo, a esta maduración bioquímica la acompañan cambios mayores en la arquitectura del pulmón. La ACTH es también importante para la diferenciación del pulmón lo que se ha evidenciado cuando se hace hipofisectomía en experimentos con animales produciéndose retardo en la maduración pulmonar (3).

\section{Efecto de los esteroides sobre la maduración pulmonar}

Mientras investigaba el mecanismo de los corticosteroides en la inducción del trabajo de parto, Liggins encontró en la década del sesenta que las ovejas paridas después de un tratamiento con corticoides a la madre, tenían unos pulmones fundamentalmente aptos antes de lo previsto por la edad gestacional. Esto ponía claramente de manifiesto que la terapia corticoidea podía suponer una manera de acelerar la madurez pulmonar fetal.

El primer ensayo clínico de esteroides para prevenir el SDR lo hicieron Liggins y Howie en 1971 (4). A un grupo de 268 mujeres entre las 24 y 36 semanas de gestación con trabajo de parto prematuro o a quienes había que terminar el embarazo antes del término se les daba el azar $12 \mathrm{mg}$. de betametasona dos veces con 24 horas de diferencia, o un placebo. Encontraron que el $4,3 \%$ de las expuestas a betametasona desarrollaron SDR contra $24 \%$ del grupo control.

En los doce años siguientes se hicieron numerosos estudios de este tipo, algunos controlados y otros no y la mayoría estuvieron de acuerdo en que los corticosteroides administrados a la madre antes del parto prematuro eran efectivos para reducir la incidencia del SDR en el niño.

El mecanismo exacto por el cual los esteroides reducen la frecuencia del síndrome de dificultad respiratoria no ha sido aclarado. Hay dos hipótesis propuestas:

1. Los esteroides inducen la producción aumentada de las enzimas responsables de la síntesis de lecitina y parece que las más importantes son las reacciones de acylación que dan origen a las lecitinas saturadas.

La actividad enzimática es máxima a las seis horas de la inyección y desciende a los niveles control aproximadamente a las 24 horas. Sin embargo, el tiempo de latencia necesario para que la cantidad total de lecitina 
alcance unos niveles que permitan una acción adecuada a nivel de la tensión superficial alveolar es de 48 horas.

2. Los esteroides tienen efecto sobre el colágeno y la elastina del pulmón. El aumento de la capacidad pulmonar que se ve después del tratamiento con esteroides puede ser entonces debido también a cambios estructurales en el pulmón, acelerando su desarrollo funcional.

La acción protectora de los esteroides parece ser transitoria. Liggins y Howie (1974) observaron que la frecuencia de dificultad respiratoria aumentaba cuando el niño nacía más de siete días después del tratamiento con betametasona, en comparación con otros niños nacidos entre uno y siete días después de haber completado el tratamiento. Por otro lado, Brown y Cols. (1979) observaron que en fetos de oveja cateterizados crónicamente, el incremento del tensoactivo consecutivo a la administración de dexametasona era transitorio, con niveles que descendían hasta los valores anteriores al tratamiento en el plazo de ocho a diez días.

\section{Efectos adversos de los esteroides:}

Es necesario puntualizar que todas las acciones sobre el feto y el recién nacido evidenciadas por los distintos autores, han sido casi en su totalidad en el animal de experimentación. Se ha hablado entonces que debido al efecto que tienen sobre el tejido conectivo podrían llevar al envejecimiento placentario, disminución del crecimiento del cerebro y pulmón y degeneración celular de los islotes pancreáticos (1). Conejos y ratas tratadas con glucocorticoides al final del embarazo tuvieron un número grande de fetos muertos (5). Un experimento en el mono Rhesus en 1978 mostró que el perímetro cefálico, el peso placentario, el peso del timo y la suprarrenal eran significativamente menores en los tratados con esteroides que en el grupo control (6). Otro experimento hecho en curíes encontró que la administración de dexametasona disminuía la producción de DNA en los hemisferios cerebrales, el cerebelo y la médula además de la reducción en los pulmones, riñones, corazón y adrenales fetales. A los catorce días después del tratamiento se normalizaba la producción de DNA pero se desconocen los efectos a largo plazo que pudieran tener estas alteraciones en la multiplicación celular dentro del SNC (6).

No obstante la variedad de secuelas en el feto y neonato de animales tratados con glucocorticoides, no se han documentado efectos adversos sobre el desarrollo en humanos.

Se ha encontrado aumento significativo de la frecuencia de hipoglicemia neonatal en los niños que recibieron betametasona in útero pero no hay resultados definitivos sobre los efectos a largo plazo de los niños sometidos a este tratamiento antes de nacer.

El 85 por ciento de los sobrevivientes del trabajo original de Liggins y Howie fueron evaluados a los cinco y siete años para documentar los efectos adversos de la betametasona antenatal. No encontraron diferencias en los test de inteligencia, vocabulario, percepción visual y madurez social entre los niños expuestos a esteroides y los controles. Tampoco hubo diferencias en el desarrollo del lenguaje, aprendizaje o problemas médicos (7).

\section{Efecto de la ruptura prematura de membranas (RPM) sobre la maduración pulmonar}

Desde 1970, múltiples grupos han examinado la relación entre RPM pretérmino y el SDR encontrando en la mayoría un efecto beneficioso de un período de latencia prolongado para reducir la frecuencia de éste.

Mediciones de $\mathrm{L} / \mathrm{S}$ o de fosfatidilglicerol en pacientes con más de 24 horas de RPM pretérmino mostraban relación L/S madura y aparición de fosfatidilglicerol a una edad gestacional más temprana. Varios grupos hicieron mediciones del cortisol en sangre materna y fetal y en líquido amniótico de pacientes con RPM encontrando mayor concentración de éste en las pacientes que tenían más tiempo de latencia y con niveles mayores de lo esperado para la edad gestacional.

En un estudio prospectivo Garite y cols. obtuvieron líquido amniótico en treinta mujeres con RPM entre 2835 semanas y encontraron que la mitad tenían relación L/ $\mathrm{S}^{3}$ 1,8:1 y ninguno de estos niños presentó SDR (8).

El estudio prospectivo más grande que existe usando medición de fosfatidilglicerol en el líquido amniótico obtenido de vagina es el de Brame y Mac Kenna en 1983 (9). En este trabajo encontraron que el fosfatidilglicerol estaba presente en el grupo de 32-35 semanas en el 25\% de las pacientes y en el $10 \%$ en el de menos de 32 semanas después de un período de latencia de una a doce horas.

Cuando se hacía nueva medición a los cuatro o cinco días, el $27 \%$ alcanzaban madurez sin el uso de esteroides o betamiméticos.

Todos estos estudios parecen pues indicar que con la RPM pretérmino ocurre una acelaración de la aparición del surfactante en el líquido amniótico antes del tiempo esperado para la edad gestacional. Inclusive cuando el análisis inicial del líquido amniótico muestra inmadurez, la maduración realmente ocurre en una forma acelerada después de la RPM en el $50 \%$ de los pacientes.

El efecto beneficio de la RPM podría deberse a una conversión más rápida de la cortisona inactiva a cortisol activo por el amnios en presencia de RPM.

\section{Efecto de los esteroides en pacientes con ruptura prematura pretérmino}

Sobra participar que es grande la controversia que existe en este aspecto. Hay dos interrogantes grandes:

1. ¿Cuándo hay ruptura de membranas pretérmino, los esteroides tienen algún efecto adicional para inducir la maduración pulmonar?

2. ¿El uso de esteroides cuando hay ruptura de membranas aumenta la frecuencia de infección materna o fetal?

Con el objeto de tener algunos elementos de juicio que permitan obtener conclusiones se hará un resumen de los principales trabajos que se han hecho en los últimos años teniendo en cuenta sólo los que se hicieron al azar y bien controlados.

\section{Nelson y cols. (1985) (10)}

Analizaron 68 pacientes con edad gestacional de 2834 semanas y los dividieron en tres subgrupos que se manejaban así: 
1. Betametasona y parto a las 48 horas del inicio de esteroides.

2. Placebo y parto a las 48 horas.

3. Placebo y manejo expectante.

Encontraron que el SDR fue de 45,50 y 33 por ciento respectivamente. La diferencia no fue significativa entre el uso y no uso de esteroides, pero llama la atención que el grupo de manejo expectante, el cual tuvo un período de latencia mayor, fue el que menor porcentaje de SDR tuvo.

La sepsis neonatal fue mayor significativamente en el grupo que utilizó esteroides; la fiebre materna antes del parto fue igual y la infección materna postparto no se evaluó.

\section{Lams y cols. (1985) (11)}

Estudiaron 73 pacientes entre las 28 y 34 semanas y las dividieron en dos grupos:

1. Hidrocortisona $500 \mathrm{mg}$ IV cada ocho horas por cuatro dosis y parto a las 48-72 horas del inicio de éste.

2. Expectante sin esteroides ni tocolíticos y parto cuando iniciaba trabajo de parto espontáneamente o había infección o sufrimiento fetal.

El SDR fue de 26,3 y 34,3 por ciento respectivamente sin significancia estadística.

La frecuencia de endometritis fue mayor en las pacientes a quienes se les aplicó esteroides pero sólo a las que se les practicaba cesárea. No hubo diferencia significativa en la frecuencia de infección neonatal (10,5 VS 8,6 por ciento).

\section{Garite y cols. (1981) (8)}

Evaluaron 160 pacientes entre las 28 y 334 semanas divididas en dos grupos:

1. Betametasona y parto a las 48 horas.

2. Expectante y parto cuando iniciaba trabajo de parto espontáneamente, había infección o sufrimiento fetal. Equipararon por edad materna, edad gestacional, período de latencia y actividad uterina. El SDR no tuvo diferencia significativa (17 VS $21 \%$ respectivamente).

Hubo tendencia a disminuir el SDR después de 48 horas de membranas rotas en ambos grupos.

Hubo más frecuencia de endometritis en las que se usó esteroides, pero cuando el parto era vía vaginal (usaron profilaxis sólo en el grupo de cesárea).

No hubo diferencia significativa en la infección neonatal aunque los cuatro casos de sepsis fueron del grupo de esteroides.

Lo trabajos que siguen incluyeron pacientes con trabajo de parto prematuro con y sin RPM, se hará referencia sólo al grupo con RPM.

\section{Schmidt y cols. (1984) (12)}

En 62 pacientes con RPM pretérmino entre las $26 \mathrm{y}$ 32 semanas se evaluó el efecto de hidrocortisona, metilprednisolona y betametasona sobre la maduración pulmonar. No se encontró diferencia significativa en la frecuencia del SDR pero a diferencia de la mayoría de los trabajos que han evaluado el uso de esteroides en pacientes con membranas íntegras, éste no encontró diferencia significativa tampoco en el grupo de membranas íntegras.

La hidrocortisona y la metilprednisolona no modificaron en nada el SDR con respecto al control.

La infección materna no aumentó significativamente en el grupo de RPM y esteroides. La infección neonatal no se evaluó específicamente para RPM.

\section{Grupo colaborativo de terapia esteroidea antenatal (1981) (13)}

Encontró en 74 pacientes con RPM pretérmino que la dexametasona no disminuía en forma significativa la incidencia del SDR con respecto al grupo placebo: 9,8 VS 12,6.

No discriminó la frecuencia de infección en el grupo de RPM pero en el grupo en general no se encontró mayor infección postparto con el uso de esteroides ni en madres ni en neonatos.

\section{Taeusch y cols. (1979) (14)}

Encontró en 81 pacientes con RPM y edad gestacional menor de 33 semanas, que la dexametasona no disminuía en forma significativa el SDR con respecto al placebo (29\% VS 30\%). Sin embargo la severidad del SDR era menor en el grupo que utilizó esteroides.

La frecuencia de infección si fue mayor en el grupo de esteroides tanto en la madre como en el neonato (madre: $27 \%$ VS $12 \%$; niño: $13 \%$ VS $6 \%$ ) cuando las membranas estuvieron rotas más de 48 horas.

\section{Schutte y cols. (1980) (15)}

Comparó la frecuencia de SDR en pacientes entre las 26 y 33 semanas manejadas con orciplenalina y betametasona con pacientes manejadas con orciprenalina y placebo, tanto con membranas íntegras como rotas, pero al evaluar sólo las pacientes con membranas rotas que tuvieron su parto entre las doce horas y los siete días de la aplicación del esteroide, el número fue muy pequeño (11 VS 13 pacientes) con 0 y $31 \%$ de SDR respectivamente, lo cual no permite obtener conclusiones objetivas. No se mencionó la frecuencia de infección.

En resumen: de los ocho trabajos que sustentan el no uso de esteroides en RPM sólo tres evaluaron exclusivamente las madres con RPM. En los tres hubo una disminución leve del SDR con el uso de esteroides aunque no alcanzó significancia estadística.

No hubo diferencia significativa en la frecuencia de infección neonatal pero de todas formas se observó un leve aumento de la frecuencia de infección neonatal con el uso de esteroides.

Sobre la frecuencia de infección materna no hubo claridad pues en uno no se evaluó, en otro hubo más endometriosis pero sólo en cesárea y en otro más endometriosis, pero sólo en parto vaginal atribuyéndose ésta al uso o no de antibióticos profilácticos.

Los otro cinco trabajos de los ocho que sustentan el no uso de esteroides, tuvieron en cuenta las pacientes con membranas íntegras también; por lo tanto al referirse exclusivamente a las pacientes con membranas rotas, sólo dos quedan con un número adecuado de pacientes. 
En estos dos aunque no es significativa la diferencia, se observa una leve diminución del SDR en el grupo de esteroides. El único que evaluó infección encontró aumento significativo de la infección materna y fetal.

Refirámonos ahora a los trabajos que si han encontrado diferencia significativa en la disminución del SDR con el uso de esteroides en la RPM:

\section{Morales y cols. (1986) (16)}

Se refirió exclusivamente a las pacientes con ruptura prematura de membranas entre las 28 y 33 semanas obteniendo 250 pacientes en total. Las dividió en dos grupos así: 121 pacientes con dexametasona y 129 pacientes con placebo.

En ambos grupos se hizo manejo expectante después de terminadas las dosis de maduración o las 48 horas de membranas rotas. Si a los siete días no habían tenido el parto, se repetía la dosis de esteroides. Se usó tocólisis si era necesario mientras se completaba el tiempo de maduración y se evaluó fosfatidilglicerol en vagina antes de iniciar el manejo.

El período de latencia de ambos grupos fue más o menos similar.

Encontraron que el SDR disminuyó en forma significativa con el uso de esteroides (25\% vs $51 \%$ ).

La frecuencia de amnionitis o sepsis neonatal fue igual y no evaluaron la endometriosis.

\section{Young (1980) (17)}

Evaluó 113 pacientes entre las 27 y 33 semanas los cuales dividió en dos grupos: 51 pacientes con dexametasona y 62 pacientes con placebo.

El embarazo se terminó en ambos grupos a las 48 horas de la ruptura encontrando que el SDR fue menor en forma significativa en el grupo en el que se utilizó esteroides (11\% VS 38\%).

La infección materna o neonatal no aumentó con el uso de esteroides.

Llamó la atención que en ambos grupos fue menor el SDR después de 24 horas de membranas rotas.

\section{Papageorgiou (1979) (18)}

Incluyó tanto pacientes con R.P.M. como membranas íntegras entre las 25 y 34 semanas y al evaluarlas solo con ruptura de membrana obtuvo 36 pacientes. Se dividieron en dos grupos (betametasona y placebo) y se hizo manejo expectante posterior a la maduración pulmonar. Se encontró que el SDR disminuyó significativamente en el grupo con esteroides (23,5\% VS 58\%) además de que la severidad del SDR cuando ocurrió, fue menor en el grupo con esteroides. No hubo diferencia en la frecuencia de infección intrauterina.

\section{Block (1977) (19)}

Dividió 69 pacientes con RPM en tres grupos a los que manejó con betametasona, metilprednisolona $y$ placebo encontrando que el SDR fue de $12 \%, 22 \%$ y $19,2 \%$, lo cual no fue significativo. No mencionó la relación con infección.

\section{Morales (1989) (20)}

Estudió 165 pacientes con RPM entre las 26 y las 34 semanas, las cuales dividió en cuatro grupos:

1. Grupo control - manejo expectante

2. Betametasona que se repetía a la semana.

3. Manejo expectante más ampicilina 2 gr cada seis horas mientras llegaba el resultado del cultivo del líquido amniótico hecho al ingreso.

4. Betametasona y ampicilina.

No se usó tocólisis y se evaluó la relación L/S en líquido amniótico de vagina o el obtenido por amniocentesis cuando era posible excluyendo del estudio las que no tenían L/S evaluada.

Encontró que los dos grupos que se manejaron con esteroides tuvieron disminución significativa del SDR (26\% VS 53\%), menor tiempo en el ventilador, menor incidencia de displasia broncopulmonar y menor severidad de hemorragia intra-ventricular. No hubo diferencia en la infección intraamniótica o sepsis neonatal.

El uso de ampicilina redujo en forma significativa el riesgo de sepsis neonatal ( $5 \%$ VS 10\%).

La conclusión de este trabajo fue con el uso de esteroides y ampicilina que se puede mejorar el resultado perinatal del niño prematuro con RPM y una L/S inmadura comprobada sin aumentar la morbilidad del feto o de la madre.

\section{Kuhn (1982) (21)}

Evaluó 216 pacientes que sometió a betametasona o placebo y manejo expectante posterior en todos. Encontró una disminución significativa del SDR con el uso de esteroides (7\% VS 25\%), la sepsis materna fue igual y la sepsis fetal no varió significativamente (19\% VS 13\%).

\section{Resumen}

Si nos basamos en la significancia estadística de los trabajos en forma individual, la mayoría no encuentran disminución significativa del SDR. Sin embargo, vimos que el número de pacientes fue escaso en varios de estos trabajos y que siempre hubo menor frecuencia (así fuera no significativa) del SDR en el grupo con esteroides 10 que podría hacer suponer que aumentando el número de pacientes podría obtenerse la significancia estadística requerida.

En cuanto al riesgo de infección de todos los trabajos sólo tres encontraron mayor frecuencia de infección en la madre antes o después del parto; los demás, o no evaluaron este aspecto o no encontraron diferencia importante. Lo mismo sucedió con la infección neonatal: sólo dos mostraron diferencia significativa en el aumento de la frecuencia de infección con el uso de esteroides.

\section{Conclusiones}

1. La tendencia es a demostrar que la frecuencia del SDR si disminuye con el uso de esteroides en los niños prematuros aún con la existencia de membranas rotas.

2. En humanos no se ha demostrado que los esteroides tengan efectos adversos en el feto o neonato. 
3. El riesgo de infección parece no aumentar en forma significativa ni en la madre ni en el neonato, pero si hay una tendencia a ser mayor cuando se usan esteroides y hay membranas rotas.

4. No se conoce a ciencia cierta el efecto a largo plazo en el niño o en el adulto sometido al uso de esteroides antenatales.

5. Teniendo en cuenta los dos riesgos, el de SDR y el de infección, podríamos hacernos las siguientes preguntas:

¿Qué es menos malo, tener un neonato conectado al ventilador, o un neonato con manejo antibiótico por una infección?
¿Es más fácil manejar y dejar menos secuelas en una endometritis, o un bebé con una membrana hialina?

De todas formas hay aún muchas cosas que no se conocen y por lo tanto lo ideal sería no utilizar en forma indiscriminada los esteroides en todas las madres que rompen membranas entre las 26 y las 34 semanas, sino en las que tengan valores inmaduros de las pruebas de maduración pulmonar o en las que no sea posible efectuar estas pruebas.

\section{BIBLIOGRAFIA}

1. Winer S., and Weinstein L. Fetal pulmonary maturity and antenatal diagnosis of respiratory distress syndrome. Obst. and Gynecol. Survey, 1987; 42(2): 75-81.

2. Cabero L. Perinatología Tomo I, Salvat Editores, Barcelona, España 1986; p. 95.

3. Pritchard J., Mac Donald P., Gant N. Williams. Obstetricia. Salvat Editores, Barcelona, (España) tercera edición; 1986.

4. Liggins GC and Howie. A controlled trail of antepartum glucocorticoid treatment for prevention of RDS in premature infants. Pediatrics, 1974; 54: 724.

5. Taeusch HW. Glucocorticoid prophylaxis for respiratory distress syndrome: A review of potential toxicity. Pediatrics 1975; 87(4): 617-623.

6. Johnson J., Mitzner W., London W et al. Betamethasone and the rhesus fetus; multisystemic effects. Am. J. Obstet. Gynecol. 1974; 133(6): 677-683.

7. Coustan D. Clinical aspects of antenatal enhacement of pulmonary maturation. Clinics in perinatology, 1987; 14(3): 697-710.

8. Garite T., Freeman R., Linzey $M$ et al. Prospective randomized study of corticosteroids in the management of premature rupture of the membranes and the premature gestation. Am. J. Obstet. Gynecol. 1981; 141(3): 508-515

9. Blackmon L., Alger L., Crenshaw C. Fetal and Neonatal outcomes associated with Premature rupture of the membranes. Clinical Obst. and Gynecol. 1986; 29(4): 797-798.

10. Nelson L., Meis P., Hatjis C et al. Premature rupture of membranes. A prospective, randomized evaluation of steroids, latent phase and expectant management, Obstet. Gynecol. 1985; 66(1): 55-58.

11. Iams J., Talbert M., Barrows H. Management of preterm prematurely ruptured membranes: A prospective randomized comparison of observation versus use of steroids and timed delivery. Am. J. Obstet. Gynecol. 1985; 151(1): 32-38.

12. Schmidt P., Sims M., Strass SH et al. Effect of antepartum glucocorticoid administration upon neonatal respiratory distress syndrome and perinatal infection. Am. J. Obstet. Gynecol. 1984; 148(2): 178-186.

13. Collaborative group on antenatal steroid therapy. Effect of antenatal dexametasone administration on the prevention of respiratory distress syndrome. Am. J. Obstet. Gynecol. 1981; 141(3): 276-286.

14. Taeusch W., Frigoletto F., Kitz MJ et al. Risk of respiratory distress syndrome after prenatal dexamethasone treatment. Pediatrics, 1979; 63(1): 64-72.
15. Schutte MF., Treffers PE., Kope JG. The influence of betamethasone and orciprenaline on the incidence of respiratory distress syndrome in the newborn after preterm labour. British Journal of Obstet. and Gynecol. 1980; 87: 127-131.

16. Morales W., Diebel D., Lazar A et al. The effect of antenatal dexamethasone administration on the prevention of respiratory distress syndrome in preterm gestations with premature rupture of membranes. Am. J. Obstet. Gynecol. 1986; 154(3): 591-595.

17. Young B., Klein S., Katz M et al. Intravenous dexamethasone for prevention of neonatal respiratory distress. A prospective controlled study. Am. J. Obstet. Gynecol. 1980; 138(2): 203-209.

18. Papageorgiou A., Desgranges M., Masson M et al. The antenatal use of betamethasone in the prevention of respiratory distress syndrome: A controlled double-blind study. Pediatrics 1979; 63(1): 73-79.

19. Block M., Kling R., Crosby W. Antenatal glucocorticoid therapy for the prevention of respiratory distress syndrome in the premature infant. Obstet. Gynecol. 1977; 50(2): 186-190.

20. Morales W., Angel J., O'Brien W et al. Use of ampicillin and corticosteroids in premature rupture of membranes: A randomized study. Obstet. Gynecol. 1989; 73(5): 721-726.

21. Kuhn RJ., Speirs AL., Pepperell RJ et al. Betamethasone, Albaterol and risks. Obstet. Gynecol. 1982; 60(4): 403-408.

22. Cotton D., Hill L., Strassner $\mathrm{H}$ et al. Use of amniocentesis in preterm gestation with ruptured membranes. Obstet. Gynecol. 1984; 63(1): $38-43$.

23. Doran TA., Swyer P., Mac Murray B et al. Results of a double-blind controlled study on the use of betamethasone in the prevention of respiratory distress syndrome. Am. J. Obstet. Gynecol. 1980; 136(3): 313-319.

24. Duff P. Premature rupture of membranes. Clinical Obst. and Gynecol. 1991; 34(4): 683-793.

25. Hallak M. and Buttoms S. Accelerated pulmonary maturation from preterm premature rupture of membranes. A myth. Am. J. Obstet. Gynecol. 1993; 169(4): 1045-1049.

26. Johnsons J., Daikoku N., Niebyl J et al. Premature rupture of the membranes and prolonged latency. Obstet. Gynecol. 1981; 57(5): 547 556.

27. Lewis N. Premature rupture of membranes. A prospective randomized evaluation of steroids, latent phase and expctant management. Obstet. Gynecol. 1985; 66: 55-58.

28. Mead P. Management of the patient with premature rupture of membranes. Clinics in Perinatology, 1980; 7(2): 243-255. 